\title{
Personalized cardiac surgery: Can noninvasive flow imaging lead to individualized resection strategies for bicuspid aortopathy?
}

\author{
Nicholas D. Andersen, MD, ${ }^{\mathrm{a}}$ and Joseph W. Turek, MD, $\mathrm{PhD}^{\mathrm{b}}$
}

\footnotetext{
From the aDepartment of Cardiac Surgery, Boston Children's Hospital, Harvard Medical School, Boston, Mass; and ${ }^{\mathrm{b}}$ Division of Cardiovascular and Thoracic Surgery, Duke University Medical Center, Durham, NC. Disclosures: Authors have nothing to disclose with regard to commercial support.

Received for publication June 17, 2018; revisions received June 17, 2018; accepted for publication June 18, 2018; available ahead of print Aug 11, 2018.

Address for reprints: Nicholas D. Andersen, MD, Department of Cardiac Surgery, Boston Children's Hospital, 300 Longwood Ave, Bader 273, Boston, MA 02115 (E-mail: Nicholas.Andersen@ cardio.chboston.org).

J Thorac Cardiovasc Surg 2018;156:2110-1 $0022-5223 / \$ 36.00$

Copyright $(c) 2018$ by The American Association for Thoracic Surgery https://doi.org/10.1016/j.jtcvs.2018.06.060
}

Bicuspid aortic valve (BAV) and BAV aortopathy represent a diverse and heterogenous group of congenital heart malformations with unique patterns of valve cusp morphology, aortic dilation, and mechanisms of disease pathogenesis and progression (Figure 1). ${ }^{1}$ However, current guidelines for BAV aortopathy do not differentiate between disease subgroups and instead recommend replacement of the proximal aorta based only on aortic diameter thresholds. ${ }^{2}$ Is this 1size-fits-all approach the ideal way to risk stratify patients with BAV?

Bollache and colleagues ${ }^{3}$ build on previous work studying the contribution of altered valve-mediated flow dynamics to aortopathy in patients with BAV. Previous studies from this group have shown that altered hemodynamic flow patterns in the proximal aortas of patients with BAV lead to increased wall shear stress (WSS), which in turn is associated with elastic fiber degradation. ${ }^{4}$ In the present study, ${ }^{3}$ a more comprehensive analysis was performed exploring the association between WSS as a continuous variable and elastic fiber thickness in resected BAV aortic tissue specimens from 27 patients. Results showed that the magnitude of proximal aorta WSS correlated with the magnitude of elastic fiber thinning, primarily in patients with BAV with aortic stenosis and those with smaller aortic diameters $(<4.5 \mathrm{~cm}){ }^{3}$ The finding that WSS correlated with elastic fiber thinning in patients with aortic stenosis is intuitive because patients with aortic regurgitation are generally believed to display more of a hereditary connective tissue disease phenotype with predominant root dilation and a lower contribution of flow mechanics to aortopathy. However, the finding that WSS correlated with elastic fiber thinning in patients with aortic diameter $<4.5 \mathrm{~cm}$ is somewhat puzzling. However, these results may be explained by selection bias. Given that all patients in the series underwent aortic replacement, it is possible that patients with smaller aortas had more aortic risk factors or evidence of rapidly

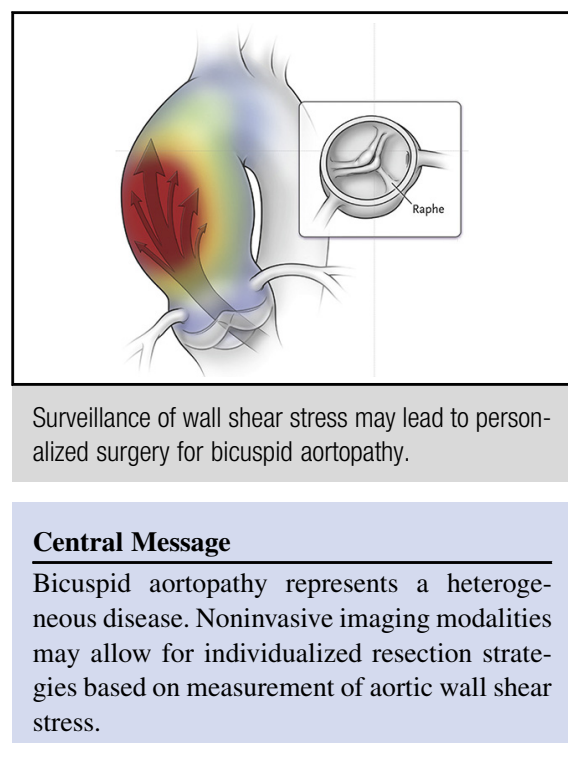

See Article page 2112.

progressive disease and were intentionally selected for aortic replacement at smaller diameters.

Overall, the study by Bollache and colleagues ${ }^{3}$ provides further evidence that valve-mediated flow alterations contribute to the pathogenesis of BAV aortopathy in some patients and suggests that measurement of WSS could serve as a noninvasive biologic marker of disease severity, or stability, to assist with surgical decision making. However, several limitations of the study prevent any robust clinical conclusions at this time. The study was underpowered to determine whether valve cusp morphology, or severity of valvular stenosis or regurgitation, influenced the association between WSS and elastic fiber thickness, precluding more granular patient subgroup risk stratifications. More importantly, correlations between WSS and the natural history of BAV aortopathy are not known. A longitudinal analysis with serial imaging tests is needed to determine whether aortic wall areas under the greatest WSS are prone to accelerated degeneration, dilation, rupture, or dissection. These observations would need to be evaluated in relation to standard aortic diameter measurements to determine whether there is any superiority to using WSS to guide surgical decision making. Ideally, investigators would be able to identify a critical WSS threshold that could serve as an 


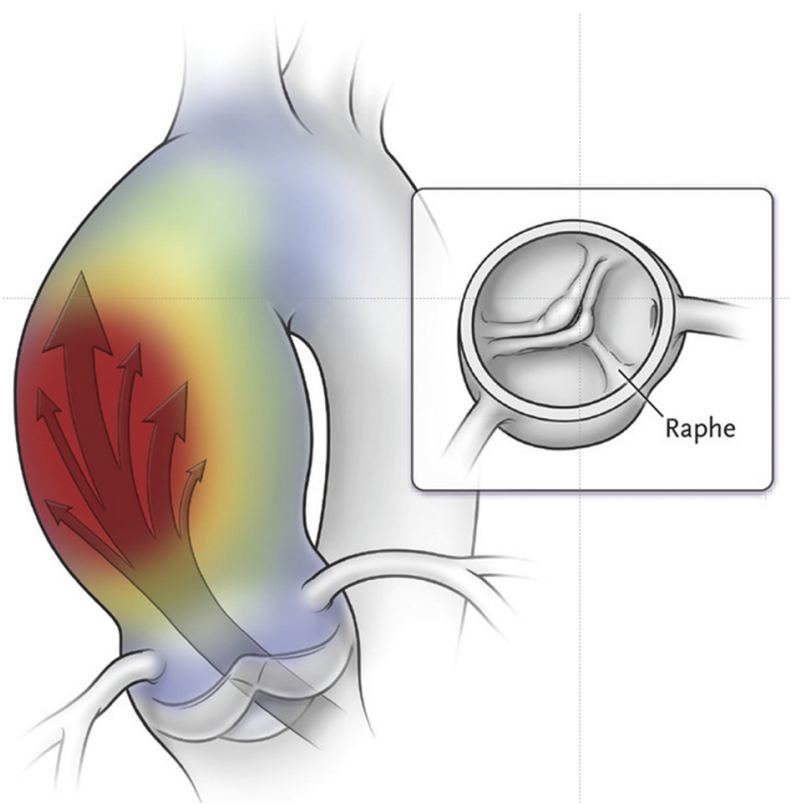

FIGURE 1. Surveillance of wall shear stress may lead to personalized surgery for bicuspid aortopathy. independent trigger for aortic replacement irrespective of aortic diameter.

Despite these limitations, the study by Bollache and colleagues ${ }^{3}$ provides important incremental knowledge regarding the pathogenesis of BAV aortopathy, and holds promise for the development of individualized, biologically based predictors of disease progression that can help to identify patients at greatest risk of aortic events or those who may benefit from earlier or later surgery.

\section{References}

1. Verma S, Siu SC. Aortic dilatation in patients with bicuspid aortic valve. $N$ Engl J Med. 2014;370:1920-9.

2. Hiratzka LF, Creager MA, Isselbacher EM, Svensson LG, Nishimura RA, Bonow RO, et al. Surgery for aortic dilatation in patients with bicuspid aortic valves: a statement of clarification from the American College of Cardiology/ American Heart Association Task Force on Clinical Practice Guidelines. J Am Coll Cardiol. 2016;67:724-31.

3. Bollache E, Guzzardi DG, Sattari S, Olsen KE, Di Martino ES, Malaisrie SC, et al. Aortic valve-mediated wall shear stress is heterogeneous and predicts regional aortic elastic fiber thinning in bicuspid aortic valve-associated aortopathy. J Thorac Cardiovasc Surg. 2018;156:2112-20.e2.

4. Guzzardi DG, Barker AJ, van Ooij P, Malaisrie SC, Puthumana JJ, Belke DD, et al Valve-related hemodynamics mediate human bicuspid aortopathy: insights from wall shear stress mapping. J Am Coll Cardiol. 2015;66:892-900. 